\title{
Conflictividad social en torno a los azudes del Júcar a finales del siglo XVI. Un problema recurrente por la gestión del agua
}

\author{
Frederic Aparisi Romero ${ }^{1}$ y Daniel Muñoz Navarro ${ }^{2}$ \\ ${ }^{1}$ Universitat de València. ${ }^{2}$ Università degli Studi di San Marino - Universitat de València \\ Frederic.aparisi@uv.es, Daniel.munoz@uv.es
}

\begin{abstract}
Resumen: El aprovechamiento de los recursos hídricos del río Júcar ha sido un elemento de conflicto recurrente a lo largo de la historia. No obstante, en algunos momentos esta conflictividad se agudizaba. Es el caso de la etapa comprendida entre finales del siglo XVI y los primeros años del XVII, coincidiendo con las variaciones climáticas derivadas de la Pequeña Edad Glacial. La construcción de paradas, azudes, motas u otros elementos que podían frenar el drenaje del curso hídrico del río produjo un choque de intereses (económicos, políticos, jurisdiccionales...) entre las diferentes villas de la Ribera del Júcar (Carcaixent contra Alzira, Alzira contra Sueca...), canalizados en buena medida a través de la vía judicial. En este estudio pretendemos analizar el rastro documental generado en estos años de incremento de las riadas y las inundaciones, concentrando nuestra atención en la conflictividad generada en torno a los azudes del Júcar.
\end{abstract}

Palabras clave: Conflictividad, azudes, pleitos, Ribera del Júcar, Siglo XVI

\begin{abstract}
The uses of water resources of the river Júcar have been a recurring element of conflict throughout the history that, at some times, deepened. This is what happened between the late sixteenth century and early seventeenth centuries, coinciding with climatic variations derived from the Little Ice Age. The building of dams, specks and other structures to slow the free flow of the river produced a clash of interests in different aspects such as economic, political or jurisdictional between the different towns of the area of the Ribera del Júcar (Carcaixent versus Alzira, Alzira versus Sueca...) channelled largely through the courts of the kingdom. This paper seeks to analyse these disputes using the legal evidence and the references about the building of dams and floods that happened in this period.
\end{abstract}

Keywords: Disputes, Dams, Lawsuits, Ribera del Júcar, 16th century

\section{Introducción}

A lo largo de la historia, el aprovechamiento de los recursos hídricos del río Júcar ha sido un elemento de conflicto recurrente que, condicionado por factores diversos, ha llegado incluso hasta nuestros días. En palabras de Tomás Peris Albentosa (1985: 75-108), ya desde el siglo XVII diferentes autores coinciden en explicar las riadas como resultado complejo de una serie de rasgos naturales (características de la cuenca baja del Xúquer, anomalías climáticas, etc.) y un conjunto de actuaciones antrópicas perjudiciales (construcción de infraestructuras que afectaban al drenaje de las aguas, deforestación de la cuenca, etc. $)^{1}$.

\footnotetext{
1 Poco podemos añadir a lo ya expuesto por este autor en relación a los factores causales (antrópicos y naturales) de las inundaciones en la Ribera en el mencionado artículo. También remitimos al lector a la obra de Alberola Romà (2010).
} 
No obstante, en algunos momentos este conflicto se agudizaba, debido a un recrudecimiento de las condiciones climáticas, provocando un mayor enfrentamiento social y judicial de las poblaciones afectadas. Este es el caso de la etapa comprendida entre finales del siglo XVI y los primeros años del XVII, un momento de especial dificultad en la Ribera del Júcar, que coincide con las variaciones climáticas derivadas de la Pequeña Edad Glacial².

A través de esta comunicación pretendemos analizar el rastro documental generado en estos años de incremento de las riadas y las inundaciones, concentrando nuestra atención en la conflictividad en torno a los azudes del Júcar durante este periodo. La construcción de paradas, azudes, motas u otros elementos que podían frenar el drenaje del curso hídrico del Júcar produjo un choque de intereses (económicos, políticos, jurisdiccionales...) entre las diferentes villas de la Ribera del Júcar (Carcaixent contra Alzira, Alzira contra Sueca...), canalizados en buena medida a través de la vía judicial. El elevado número de pleitos es la expresión plausible de un conflicto irresoluble, que aún a día de hoy sigue latente (Peris Albentosa, 1985: 102-107). No obstante, fue a partir de este momento (finales del siglo XVI) cuando comenzaron a desarrollarse toda una serie de mecanismos administrativos y técnicos, que intentaron buscar, aunque con poco éxito, el origen y la solución de este conflicto ${ }^{3}$.

Estudiar estos pleitos nos ayuda a comprender mejor los factores que contribuyeron a mantener viva la conflictividad en torno a los azudes del Júcar durante toda la Edad Moderna ${ }^{4}$. Fruto de la contraposición de intereses, resulta interesante analizar como las poblaciones ribereñas mantuvieron una doble moral en relación con las paradas y azudes del Júcar, en base a su posición con respecto a ellas, rechazando la utilidad de aquellas que se situaban aguas abajo y, al mismo tiempo, defendiendo la necesidad de las paradas ubicadas aguas arriba.

\section{La Pequeña Edad Glacial y su repercusión social sobre la Ribera del Júcar}

A través de los registros documentales valencianos se puede detectar el enfrentamiento histórico por el uso del agua en torno al río Júcar ${ }^{5}$. Al menos desde la época de la conquista, se aprecia esta pugna entre los que defendían un curso fluvial abierto a la navegación (los cuales contaban inicialmente con el favor de la Corona) y los molineros, que pretendían segmentar el río para aprovechar la fuerza motriz de sus aguas ${ }^{6}$. Ferran I, por medio del privilegio de 26 de mayo de 1415, legisló a favor de estos últimos, disponiendo que pudieran mantener las compuertas de sus azudes cerradas, con la condición de tener la obligación de abrirlas en el momento en que tuviesen que pasar embarcaciones o maderadas procedentes de aguas arriba. No obstante, este conflicto secular fue cambiando, de manera paralela a la transformación experimentada por el territorio que circundaba el Júcar, adaptándose a los nuevos tiempos. La progresiva difusión del regadío (y la creación de un gran hidrosistema derivado de ella) avivó el

\footnotetext{
2 Probablemente, el texto que aborda con mayor amplitud este tema y la hidrogeomorfología de la región es el de Ruiz Pérez (2001).

${ }^{3}$ Mateu Bellés (1983). Además de las visuras citadas en este artículo (la de Pedro Esquivel, la de Vicent Vicens y la de Pau de Racsas), existieron visuras de menor escala derivadas de los pleitos sucesivos, como la recogida en uno de los pleitos que analizaremos en este trabajo, el que enfrentó a Alzira y Sueca en 1604.

4 Para obtener una visión de conjunto, véase Peris Albentosa (1997).

5 Ante la amplitud de la bibliografía relativa al regadío en la Ribera del Júcar, remitimos a la contenida en una reciente obra colectiva: Hermosilla Pla (2006).

${ }^{6}$ Sobre la conflictividad entre regantes y molineros Esquilache y Martínez (2012).
} 
enfrentamiento entre los partidarios y detractores de este nuevo sistema económico, provocando profundas repercusiones políticas, sociales, paisajísticas e incluso mentales en la Ribera del Júcar (Furió y Martinez, 1993).

Diferentes autores han definido las décadas finales del siglo XVI como un periodo de cambio climático y de incremento de las riadas e inundaciones, coincidiendo con el punto álgido de la denominada Pequeña Edad Glacial (Butzer et al., 1983; Mateu, 1989 y Ruiz Pérez, 2001). En este sentido, hace ya varias décadas que Butzer, Miralles y Mateu (1983) apuntaron a un periodo de fuertes variaciones climáticas entre 1589 y 1629; sin embargo, pensamos que ya en los años precedentes se había incrementado la conflictividad social, basándonos en la información contenida en los registros documentales. En esta línea, José Miguel Ruiz Pérez (2001) hace referencia al recrudecimiento de las condiciones climáticas desde comienzos de la década de 1580, situándose en estos años el punto más álgido de la Pequeña Edad Glacial, cuya consecuencia más plausible sobre el territorio que estamos abordando en este estudio fue un incremento significativo del número de riadas e inundaciones del Júcar. Y es que este aumento de las avenidas y desbordamientos de los ríos sería la manifestación en el área mediterránea del aumento de las nevadas y temporales con períodos de lluvia intensa más prolongados (Alberola, 2014).

Este enfriamiento generalizado tuvo una repercusión directa sobre la llanura aluvial del Júcar, aumentando no sólo la frecuencia, sino también la intensidad de las riadas y contribuyendo a acrecentar el enfrentamiento entre las poblaciones ubicadas en los márgenes del Júcar. Este hecho quedó reflejado en la documentación histórica de los principales archivos municipales de la Ribera, donde queda plasmada la mayor preocupación de las autoridades locales por regular el curso fluvial del Júcar y, en paralelo, una mayor presencia de pleitos relacionados con este conflicto recurrente, agudizado por el aumento de los desbordamientos del río (Granell, 1905-1907; Parra Ballester, 1961).

Junto a estos factores, son de gran importancia las condiciones geomorfológicas de la Ribera, caracterizada por dos planicies de inundación, una convexa, que afecta a la Ribera Alta, y otro cóncavo, el de la Ribera Baja, separados por un fuerte estrechamiento del lecho fluvial -o cono aluvial-, a la altura de Algemesí, donde se produce el desagüe del río Magro sobre el Júcar. Esto condiciona el curso de río, creando un efecto "cuello de botella", que dificulta el drenaje del agua, más aún en momentos de exceso de caudal, produciendo el efecto del "regolf', esto es, la contravenida del agua en dirección contraria a su curso?.

En este estudio, concentraremos nuestra atención en la documentación judicial, ya que es la que nos aporta una mayor riqueza de información en relación con la conflictividad recurrente en torno a los azudes del Júcar. Aportamos un primer listado, que podría ser ampliado rastreando la información existente en otros archivos valencianos, especialmente el Arxiu del Regne de València (Real Audiencia, Batlia, Governació...):

1. Proceso de la villa de Alzira contra Albalat, Polinyà y Corbera, por la construcción de una nueva parada sobre el río Júcar $(1580)^{8}$.

2. Proceso de súplica incoado por la villa de Alzira sobre la causa antigua de los azudes $(1587)^{9}$.

\footnotetext{
${ }^{7}$ De hecho, la riada de 1589 afectó sobre todo a Algemesí ya que el río Magro, o Sec, no podía desaguar en el Júcar y ello provocaba el regolfe de las aguas, que llegaron a entrar dentro de las casas. El mismo Juan de Ribera tuvo que guarecerse de estas en casa del rector de la parroquia local (Domingo Borràs, 1983).

8 Arxiu Municipal d'Alzira (AMA), Plets, 17/I-83.
} 
3. Pleito de la villa de Cullera contra la villa de Sueca, con motivo de la rotura de una mota en Cullera $(1588)^{10}$.

4. Provisio ad regimen azudes fluminis Xucar aperturarum $(1595)^{11}$.

5. Pleito de la villa de Carcaixent contra la de Alzira, por la reconstrucción del azud ubicado junto al puente de esta villa $(1598)^{12}$.

6. Proceso de la villa de Alzira contra la de Sueca, a causa de la reconstrucción de un azud en el territorio de esta última población $(1604)^{13}$.

Centraremos nuestra atención en tres de estos pleitos: el primero (1580) y los dos últimos (1598 y 1604). Esta selección responde, en primer lugar, a su distribución cronológica ya que abarca un abanico temporal de veinte y cinco años, justo en el punto más frío de la Pequeña Edad Glacial. Un segundo aspecto considerado es su diversidad geográfica, que nos ofrece diferentes posiciones y visiones respecto al río y los conflictos que se derivan. Esta disparidad pone de manifiesto la implicación de un gran número de villas y universidades de la Ribera del Júcar, tanto de la Alta como de la Baja (Vilanova de Castelló, la Pobla Llarga, Alberic, Albalat de Pardines o Polinyà del Xúquer) y explica el origen diverso de los testigos que participan en estos procesos. No obstante, una población destaca sobre el resto, tomando parte activa en la mayor parte de los pleitos recogidos en nuestro estudio, Alzira, villa de realengo y principal centro urbano y político de ambas riberas. Por último, como no podía ser de otra manera, el estado de conservación de los documentos también ha condicionado la investigación, obligándonos a restringir nuestro análisis y a prescindir de determinados documentos ante el deterioro de los mismos.

En el proceso de 1580, incoado ante el Tribunal de la Gobernación, Pedro Torres de Viver, síndico de la villa de Alzira, denunció que los lugares de Albalat de Pardines y Polinyà intentaban hacer obra nueva en el río, en la partida de Moncada. En su argumentación preconizaba que este azud causaría grandes daños a la villa real y su término en época de lluvias, en el caso de que finalmente fuese construido. En su defensa, las poblaciones de Albalat y Polinyà alegaban que su intención era la de rehacer una obra vieja, y que, en consecuencia, no podía causar daños en Alzira. Así mismo, se apuntaba que la villa de Alzira ha fabricado recientemente un azud de mampostería junto al puente de Alizira, que atraviesa todo el río [...] que es verdadera causa de las inundaciones de la villa. Este nuevo azud derivaba el agua para que dicha aygua tomara dicha villa en medio y la ayla. Finalmente, el Tribunal de la Gobernación sentenció a favor de Alzira. No obstante, aquello que más nos interesa es la recusación aportada por Albalat y Polinyà, que acusaba a Alzira de utilizar una doble moral en relación con los azudes del Júcar. (Figura 1).

No en vano, el siguiente documento que aportamos, el pleito de 1598, incide en esta misma paradoja. La instancia judicial interpuesta ante la Real Audiencia por la villa de Carcaixent en contra de Alzira se centra en la construcción de una nueva obra en el río, que según la acusación se encontraba justo en el puente de San Gregorio. Esta nueva obra tenía como objetivo principal el que el agua del Júcar discurriera por el conocido como bras real, poniendo dins les dites arcades a la part de damunt certes vigues dretes clavades fortament en lo solc [...] impedint lo curs de l'aygua de dit riu [...] regolfant dita aygua, faça depòsit de tarquim, broça $i$ arena.

9 Arxiu Municipal de Sueca, Plets, F.A. 102, llibre 299. El análisis de este documento ha sido descartado debido al pésimo estado de conservación del documento, lo cual no resulta óbice para remarcar su importancia en el contexto histórico abordado en este trabajo.

10 AMS, Plets, F.A. 102, llibre 299.

11 AMA, Plets, 17/ I, 102.

12 AMA, Plets, 17/I-108.

13 AMS, Plets, caixa F.A. 102, llibre 301. 
Carcaixent estaba convencida de que ésta era la causa del incremento del número de avenidas y los daños que éstas ocasionaban a la villa y sus alrededores. Frente a ello, Alzira alegaba que no se trataba de nueva obra, sino de una parada antigua feta per a girar l'aygua del rin al brac real, apoyando su defensa en los numeros beneficios que comportaba a dicha villa de realengo, tales como la custodia y fortificación de la ciudad y la salubridad de la misma, ya que per medi de dit braçal tenen obrament e ixida les aygües fluvials $i$ demés inmundícies dels veïns $i$ habitadors de dita vila, evitando de este modo enfermedades y mortandades. El documento no recoge la sentencia de este proceso, pero cabe suponer que nuevamente la razón le fue otorgada a Alzira, ya que el meandro que circundaba esta villa siguió sin ser estrangulado y las aguas del Júcar circundaron la ciudad hasta bien entrado el siglo XX. Nuevamente, encontramos un proceso judicial que pretendía resolver, sin éxito, un conflicto recurrente entre dos poblaciones de la Ribera del Júcar, entre las cuales Alzira ejercía una posición dominante.

Por último, en 1604, encontramos un nuevo proceso judicial, esta vez instado ante la Real Audiencia por la villa de Alcira contra la de Sueca, con el objetivo de frenar la reconstrucción del azud de esta población, situado en el término de Riola (figura 1). En este caso, Alzira alegaba que se trataba de una obra nueva, que acarrearía grandes males, debido al freno que suponía al drenaje de las aguas y el regolfe de las mismas en caso de inundación o riada. La villa de Sueca, por el contrario, defendía que dita vila puga cloure los portells que les avingudes del riu ban fet en lo açut vell, ya que, de lo contrario, esta localidad no podría extraer el agua necesaria para abastecer su sistema de regadío ni per moldre lo forment. Ante la complejidad de la cuestión y después de los sucesivos testimonios y justificaciones de cada una de las partes, se realizó una visura técnica de la parada en cuestión, llevada a cabo por tres expertos (uno por parte de Alzira, otro de Sueca y un tercero de la Real Audiencia). Una vez realizada la visura, la Real Audiencia dictó sentencia a favor de Sueca. En definitiva, la utilidad y necesidad de este azud, que abastecía de agua una creciente y fecunda agricultura de regadío en el término de esta localidad, permitiendo al mismo tiempo aprovechar la fuerza motriz de las aguas para garantizar el funcionamiento de los molinos que aseguraban el abastecimiento de harina a esta población, se impuso a la oposición interesada ejercida por las influyentes autoridades políticas de la villa de Alzira.

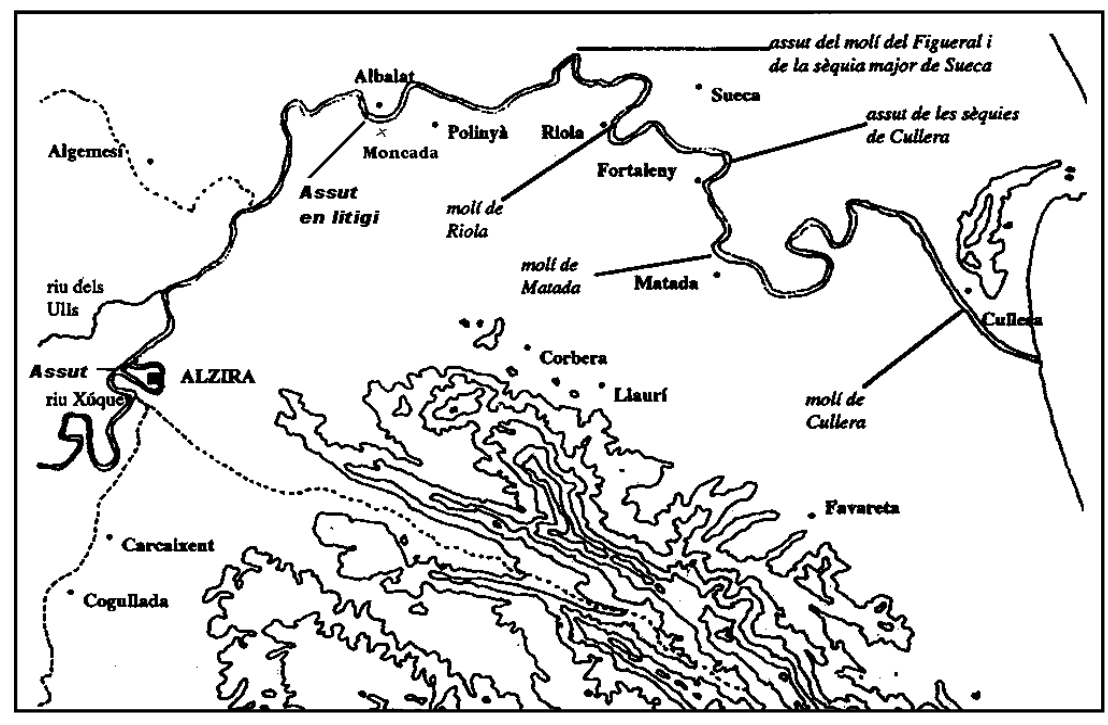

Fig. 1. Localización de los azudes y molinos del río Júcar a finales del siglo XVI (Furió y Martínez, 1993). 


\section{Gestión del agua y defensa de los intereses locales. Un problema recurrente}

Como hemos podido analizar a través de la documentación archivística y las referencias bibliográficas, los usos de las aguas del Júcar generaban, pues, un choque constante de intereses entre las diferentes villas y comunidades de la Ribera. El núcleo esencial de los conflictos giraba en torno a la forma de construcción de nuevos azudes o de reparación de los ya existentes que la fuerza del agua había destruido o mermado, puesto que lo que podía resultar provechoso para unos suponía una seria amenaza para otros.

Estos esfuerzos por imponer los intereses particulares de cada una de las poblaciones en la gestión hídrica del Júcar tenían también su lectura en clave política. No en vano, a lo largo del siglo XVI, algunos núcleos consiguieron segregarse de las villas a las que pertenecían, principalmente Alzira, y constituirse como universitas independientes estableciendo su propio ámbito jurisdiccional y su gestión del territorio. La construcción de una acequia nueva se convertía, pues, en la máxima expresión de esta autonomía y la expansión e intensificación del regadío en la Ribera a lo largo de los siglos XVI-XVIII permitió el fortalecimiento de las comunidades y de su capacidad de acción política (Ruiz Pérez, 2001).

Fruto de esta conflictividad surgían dos actitudes o posturas que se derivaban de la posición respecto al azud, distinguiendo entre aguas arriba (sobirana) o aguas abajo (jussana). Estas dos lógicas, sin embargo, no eran excluyentes ya que todas las poblaciones eran sobiranes o jussanes con respecto a otras, en función de su ubicación con respecto al azud o la parada que fuese la fuente del conflicto. Se trata, por tanto, de un doble criterio que podemos observar claramente en la documentación consultada. Ante el hecho de construir, o rehacer, una parada, los planteamientos varían según la posición con respecto a éste. Los testimonios aportados por cada una de las partes implicadas en los procesos nos permiten afirmar que los argumentos esgrimidos no respondían a una interpretación racional del conflicto (basada en criterios técnicos o empíricos sólidamente fundamentados), sino más bien a una visión interesada del problema, en la que los testimonios defendían la lógica que más les beneficiaba (a ellos y a su comunidad). De este modo, se carecía de una visión conjunta de la gestión del agua y, en consecuencia, se alimentaba la continuidad del enfrentamiento entre las poblaciones ribereñas.

Esta aparente paradoja, que hemos podido observar en los planteamientos de los síndicos expuestos anteriormente, resulta muy evidente en uno de los testimonios de los pleitos analizados en este trabajo. Los testimonios de Andreu Talens, un campesino de Albalat de Pardines, resultan paradigmáticos, ya que éste personaje tomó parte como testigo en dos de los procesos consultados, el de 1580 y el de 1604. Pese a que en ambos pleitos se dirimía una problemática muy similar (la construcción de una nueva parada en el río Júcar), sus argumentos fueron radicalmente opuestos en 1580 con respecto a los que empleó veinticuatro años después (aunque debemos tener en cuenta que también era distinta su ubicación con respecto a la nueva obra).

En el primer proceso, Alzira pleitea contra Albalat de Pardines y Polinyà del Xúquer ante la voluntad de estos dos lugares de construir un nuevo azud. Aquí, Andreu Talens se encontraba aguas abajo, en una posición jussana, y defendía la necesidad de construir el azud por los grandes beneficios que generaría a las comunidades implicadas, sin causar perjuicio alguno a las villas que se encuentran aguas arriba. Sin embargo, en 1604 Alcira litigaba contra Sueca para que esta no pudiera reconstruir el azud, destruido por las últimas inundaciones, argumentado que se trataba de una obra nueva, cuya construcción 
perjudicaba a la villa de Alzira y el resto de poblaciones situadas aguas arriba. En este proceso judicial, el campesino de Albalat se encontraba en una posición sobirana, por lo que en su alegato apoyaba que se prohibiera a la villa de Sueca reconstruir su azud, alegando el grave perjuicio que ello comportaba para las comunidades situadas por encima de la parada.

El comportamiento de Talens no fue un hecho aislado, puesto que si consultamos la lista de testimonios de los procesos analizados encontramos diversos casos similares, en los cuales el discurso había variado en relación con la posición del testimonio respecto al azud. Podemos citar dos vecinos de la villa de Alzira: Bernat Torremocha y Alexis Bonet, labrador y obrero respectivamente, los cuales testificaron en los procesos de Alzira contra Albalat y Polinyà, y también en el de Carcaixent contra Alzira. Nuevamente, los testimonios aportados por estos vecinos eran contrapuestos en uno y otro litigio. Una contradicción que sólo se explica si tenemos en cuenta la posición del testigo con respecto al conflicto y su elemento central, el azud, y la superposición de los intereses particulares al bien común que podría derivarse de una gestión colectiva de los recursos hídricos del Júcar.

No obstante, ante el elevado número de pleitos existentes en torno a esta cuestión y la pervivencia del conflicto, desde las postrimerías del siglo XVI empezaron a desarrollarse toda una serie de mecanismos administrativos y técnicos que intentan buscar el origen y la solución de este problema. En este sentido, encontramos múltiples visuras al cauce del río. Además de las citadas en este trabajo, la de Pere Esquivel, la de Vicent Vicens y la de Pau de Racsas, existieron otras visuras de menor entidad derivadas de los sucesivos pleitos como la que recoge el que enfrentó a Alzira y Sueca en 1604, con la participación de tres expertos, uno por cada una de las partes, y un tercero designado por la Real Audiencia (Mateu, 1983). Sin embargo, la participación de estos expertos, a través de visuras, y la intervención de la Real Audiencia (como antes el tribunal de la Gobernación) no llegaron a solucionarlos. No obstante, suponía un avance en el modo en que las sociedades abordaban su relación con el territorio, racionalizando (sobre una base científica cada vez más sólida) los problemas surgidos en esta dialéctica y recurriendo cada vez más al conocimiento de los expertos y a la técnica como elemento clave en la domesticación del medio y la gestión de sus recursos hídricos.

\section{Conclusiones}

En definitiva, como hemos tratado de demostrar en nuestro estudio, entre finales del siglo XVI e inicios del XVII se produjo una intensificación coyuntural de la conflictividad recurrente derivada de la gestión de los recursos hídricos del Júcar. Este acrecentamiento de las tensiones se debió principalmente a una confluencia de diversos factores ya mencionados: lluvias abundantes de forma continuada, la peculiar orografía del territorio que conforma la Ribera del Júcar (tanto la Alta como la Baja) o los intereses políticos y económicos de cada comunidad. Y es que el Júcar no puede ser considerado únicamente como un accidente geográfico, sino también como un producto histórico y cultural, resultado de la incidencia de la acción humana sobre el medio. En este sentido, a menudo los efectos perniciosos de las inundaciones nos hacen olvidar que las aguas del Júcar eran y son la principal fuente de riqueza de la Ribera del Júcar y el motor del desarrollo histórico de este territorio. 


\section{Referencias}

Alberola Romà, A. (2010): Quan la pluja no sap ploure: sequeres i riuades al Pais V alencià en l'Edat Moderna, Valencia.

Alberola Romà, A. (2014): Los cambios climáticos. La Pequeña Edad del Hielo en España, Madrid, Cátedra.

Butzer, K. W, Miralles, I. y Mateu, J. (1983): Las crecidas medievales del río Júcar según el registro geo-arqueológico de Alzira, Cuadernos de Geografia, 32-33, pp. 311-332.

Esquilache, F., Martínez, I. (2012): Les dificultats en l'aprofitament de les aigües del Xúquer. El molí, l'assut i la nora de Matada (s. XIII-XVI), Estudis d'Història Agrària no 24, p. $55-76$.

Furió, A., Martínez, L.P. (1993): Assuts i molins sobre el Xúquer en la Baixa Edat Mitjana, en Sociedades en transición: IV Congreso de Arqueología Medieval Española, tomo III, Madrid, pp. 575-586.

Granell, J. B. (1905-1907): Historia de Sueca: desde los primitivos tiempos hasta el presente, vol. I-II, Sueca.

Hermosilla Pla, J., dir., (2006): Las Riberas del Xúquer: Paisajes y patrimonio valenciano, Valencia, Generalitat Valenciana-Universitat de València.

Domingo Borràs, J.A. (1983): Les nostres riuades Per què a hores d'ara?, Berca, 19, pp. 8-20.

Mateu Bellés, J. (1983): La ciència i la técnica davant les revingudes del Xúquer (16351905): notes preliminars, Cuadernos de Geografia, 32-33, p. 243-264.

Mateu Bellés, J. (1989): Assuts i vorals fluvials regades al País Valencià medieval, en Los paisajes del agua, Universitat de València-Universitat d'Alacant, Valencia, pp.165-185.

Parra Ballester, J. Ma (1961): Catálogo del Archivo Municipal de la ciudad de Alcira, vol. I-II, Alzira.

Peris Albentosa, T. (1985): Las inundaciones del Xúquer (siglos XV-XIX), un exponente relevante de la cuestión hidráulica en tierras valencianas, Revista de Historia Moderna. Anales de la Universidad de Alicante, 23, pp. 75-108.

Peris Albentosa, T. (1997): La conflictividad hidráulica en el País Valenciano entre los siglos XIII y XVIII, Areas: Revista internacional de Ciencias Sociales, n. 17, págs. 43-60.

Ruiz Pérez, J.M. (2001): Hidrogeomorfología del llano de inundación del Júcar. Tesis doctoral dirigida por J. F. Mateu Bellés. Universitat de València, Facultat de Geografía i Història, Valencia. 